\title{
DERIVED CATEGORIES AND KUMMER VARIETIES
}

\author{
PAOLO STELLARI
}

\begin{abstract}
We prove that if two abelian varieties have equivalent derived categories then the derived categories of the smooth stacks associated to the corresponding Kummer varieties are equivalent as well. The second main result establishes necessary and sufficient conditions for the existence of equivalences between the twisted derived categories of two Kummer surfaces in terms of Hodge isometries between the generalized transcendental lattices of the corresponding abelian surfaces.
\end{abstract}

\section{INTRODUCTION}

The Kummer variety of an abelian variety $A$ is the quotient $\mathrm{K}(A):=A /\langle\iota\rangle$, where $\iota(a)=-a$ for any $a \in A$. The singular variety $\mathrm{K}(A)$ has an orbifold structure and it admits a minimal crepant resolution $\operatorname{Km}(A)$ if and only if the dimension of $A$ is 2 . In this case $\operatorname{Km}(A)$ is a $\mathrm{K} 3$ surface (i.e. it is simply connected and its dualizing sheaf is trivial) and it is called the Kummer surface of A. More generally, we can associate to the global quotient $\mathrm{K}(A)$ the smooth quotient stack $[A /\langle\iota\rangle]$.

In [11] Hosono, Lian, Oguiso and Yau proved that,

(A) given two abelian surfaces $A$ and $B, \mathrm{D}^{\mathrm{b}}(A) \cong \mathrm{D}^{\mathrm{b}}(B)$ if and only if

$$
\mathrm{D}^{\mathrm{b}}(\mathrm{Km}(A)) \cong \mathrm{D}^{\mathrm{b}}(\mathrm{Km}(B)) \text {. }
$$

Their argument runs as follows: They notice that, due to the geometric construction of the Kummer surfaces $\operatorname{Km}(A)$ and $\operatorname{Km}(B)$, the transcendental lattices of $A$ and $B$ are Hodge-isometric if and only if the transcendental lattices of $\operatorname{Km}(A)$ and $\operatorname{Km}(B)$ are Hodge-isometric. Then, they apply a deep result of Orlov which says that two abelian or K3 surfaces have equivalent derived categories if and only if their transcendental lattices are Hodge-isometric (see Theorem 2.2). From this it is evident that $(\mathrm{A})$ can be reformulated in the following way:

(B) given two abelian surfaces $A$ and $B, \mathrm{D}^{\mathrm{b}}(\mathrm{Km}(A)) \cong \mathrm{D}^{\mathrm{b}}(\mathrm{Km}(B))$ if and only if there exists a Hodge isometry between the transcendental lattices of $A$ and $B$.

Since Mukai proved in 20] that two K3 surfaces with Picard number greater than 11 and with Hodge-isometric transcendental lattices are isomorphic, (A) and (B) are equivalent to the following statement:

(C) given two abelian surfaces $A$ and $B, \mathrm{D}^{\mathrm{b}}(A) \cong \mathrm{D}^{\mathrm{b}}(B)$ if and only if $\operatorname{Km}(A) \cong \operatorname{Km}(B)$.

The aim of this paper is to address (A), (B) and (C) in two more general contexts. Our first result shows that if $A_{1}$ and $A_{2}$ are abelian varieties with equivalent derived categories, then the derived categories of the stacks $\left[A_{1} /\langle\iota\rangle\right]$ and $\left[A_{2} /\langle\iota\rangle\right]$ are equivalent as well. In fact we will prove the following:

Theorem 1.1. Let $A_{1}$ and $A_{2}$ be abelian varieties. If $\mathrm{D}^{\mathrm{b}}\left(A_{1}\right) \cong \mathrm{D}^{\mathrm{b}}\left(A_{2}\right)$, then there exists a Fourier-Mukai equivalence $\mathrm{D}^{\mathrm{b}}\left(\left[A_{1} /\langle\iota\rangle\right]\right) \cong \mathrm{D}^{\mathrm{b}}\left(\left[A_{2} /\langle\iota\rangle\right]\right)$.

Conversely, if $\mathrm{D}^{\mathrm{b}}\left(\left[A_{1} /\langle\iota\rangle\right]\right)$ and $\mathrm{D}^{\mathrm{b}}\left(\left[A_{2} /\langle\iota\rangle\right]\right)$ are equivalent, then there is an isomorphism of Hodge structures $\widetilde{H}\left(A_{1}, \mathbb{Q}\right) \cong \widetilde{H}\left(A_{2}, \mathbb{Q}\right)$.

The Hodge structures mentioned in the second part of the previous statement will be defined in Section 2.2 and the proof of this result will occupy almost all Section 3. As we will show in Section 3.3, when we deal with abelian surfaces this result leads to a direct proof of one implication in (A). 
An application to the number of birational generalized Kummer varieties is given in Proposition 3.3 .

Our second main result treats the two-dimensional twisted case. Indeed, according to (B), we prove that the twisted derived categories of two Kummer surfaces are equivalent if and only if the generalized transcendental lattices of the corresponding abelian surfaces are Hodge isometric. More precisely the result (proved in Sections 4.1 and 4.2) is as follows:

Theorem 1.2. Let $A_{1}$ and $A_{2}$ be abelian surfaces. Then the following two conditions are equivalent:

(i) there exist $\alpha_{i}$ in the Brauer group of $\mathrm{Km}\left(A_{i}\right)$ and an equivalence between the derived categories $\mathrm{D}^{\mathrm{b}}\left(\mathrm{Km}\left(A_{1}\right), \alpha_{1}\right)$ and $\mathrm{D}^{\mathrm{b}}\left(\operatorname{Km}\left(A_{2}\right), \alpha_{2}\right)$;

(ii) there exist $\beta_{i}$ in the Brauer group of $A_{i}$ such that the twisted abelian surfaces $\left(A_{1}, \beta_{1}\right)$ and $\left(A_{2}, \beta_{2}\right)$ have Hodge-isometric generalized transcendental lattices.

Furthermore, if one of these two equivalent conditions holds true, then $A_{1}$ and $A_{2}$ are isogenous.

The notations an definitions involved in the formulation of the previous result will be explained in Sections 2.2 and 4.1. We will observe that the analogues of (A) and (C) in the twisted setting are no longer true (see Remark 4.4). Nevertheless we completely generalize the results in [11] about the number of Kummer structures on K3 surfaces in the twisted context (Proposition 4.5)). A geometric example involving abelian surfaces with Picard number two is discussed.

\section{Derived categories of abelian varieties and K3 Surfaces}

In this section we recall some facts and definitions concerning the derived categories of coherent sheaves on abelian varieties and $\mathrm{K} 3$ surfaces. In the following pages $\mathrm{D}^{\mathrm{b}}(X)$ will always mean the bounded derived category of coherent sheaves on the smooth projective variety $X$ (we will also use the same notation for the bounded derived category of coherent sheaves on a smooth quotient stack according to [16]).

Suppose that $X_{1}$ and $X_{2}$ are smooth projective varieties. Let $\mathrm{D}^{\mathrm{b}}\left(X_{1}\right)$ and $\mathrm{D}^{\mathrm{b}}\left(X_{2}\right)$ be the bounded derived categories of coherent sheaves on $X_{1}$ and $X_{2}$. Orlov proved in 25 , that any equivalence $\Phi: \mathrm{D}^{\mathrm{b}}\left(X_{1}\right) \rightarrow \mathrm{D}^{\mathrm{b}}\left(X_{2}\right)$ is a Fourier-Mukai equivalence, i.e. there exists $\mathcal{E} \in \mathrm{D}^{\mathrm{b}}\left(X_{1} \times X_{2}\right)$ and an isomorphism of functors

$$
\Phi \cong \mathbf{R} p_{2 *}\left(\mathcal{E} \stackrel{\mathbf{L}}{\otimes} p_{1}^{*}(-)\right),
$$

where $p_{i}: X_{1} \times X_{2} \rightarrow X_{i}$ is the projection and $i \in\{1,2\}$ (see also [6] for a more general statement). The complex $\mathcal{E}$ is the kernel of $\Phi$ and it is uniquely (up to isomorphism) determined. We write $\Phi_{\mathcal{E}}$ for a Fourier-Mukai equivalence whose kernel is $\mathcal{E}$. In general, given $\mathcal{E} \in \mathrm{D}^{\mathrm{b}}\left(X_{1} \times X_{2}\right)$, we write $\Phi_{\mathcal{E}}$ for a functor defined as in (2.1) (notice that $\Phi_{\mathcal{E}}$ is not necessarily an equivalence).

2.1. Derived categories of abelian varieties. Assume that $A_{1}$ and $A_{2}$ are abelian varieties of dimension $d$. For $i \in\{1,2\}$, let $\mathcal{P}_{i}$ be the Poicaré line bundle on $A_{i} \times \widehat{A_{i}}$, let $\mu_{i}: A_{i} \times A_{i} \rightarrow A_{i} \times A_{i}$ be the isomorphism such that $(a, b) \mapsto(a+b, b)$ and let $\Phi_{i}:=\mu_{i *} \circ\left(\mathrm{id} \times \Phi_{\mathcal{P}_{i}}\right)$. If $\Phi_{\mathcal{E}}: \mathrm{D}^{\mathrm{b}}\left(A_{1}\right) \rightarrow \mathrm{D}^{\mathrm{b}}\left(A_{2}\right)$ is a Fourier-Mukai equivalence with kernel $\mathcal{E}$, we get the following commutative diagram:

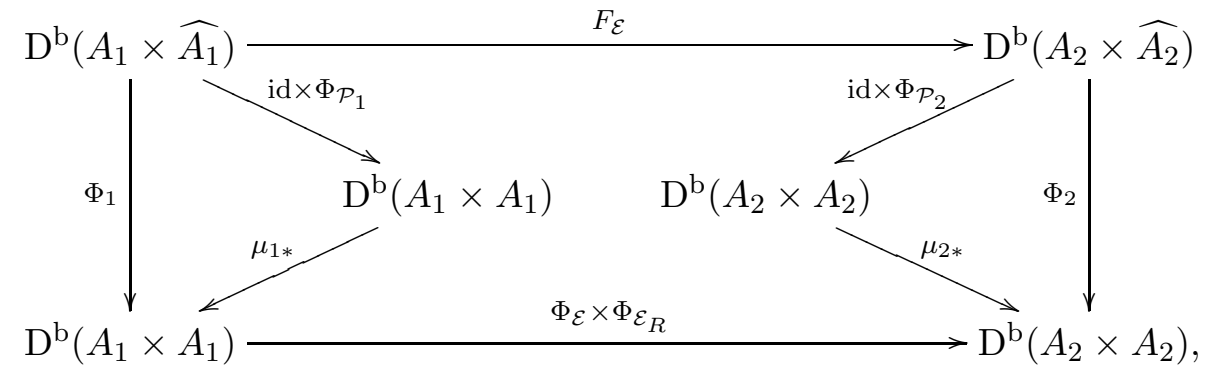


where $F_{\mathcal{E}}$ is the functor completing the diagram, $\mathcal{E}_{R}=\mathcal{E}^{\vee}[d]$ and $\Phi_{\mathcal{E}} \times \Phi_{\mathcal{E}_{R}}$ is the Fourier-Mukai equivalence whose kernel is $\mathcal{E} \otimes \mathcal{E}_{R}$. Observe that since $\Phi_{\mathcal{E}}, \Phi_{1}$ and $\Phi_{2}$ are equivalences, $\Phi_{\mathcal{E}} \times \Phi_{\mathcal{E}_{R}}$ and $F_{\mathcal{E}}$ are equivalences as well.

For $i \in\{1,2\}$, the Künneth formula yields a decomposition

$$
H_{1}\left(A_{i} \times \widehat{A_{i}}, \mathbb{Z}\right) \cong H_{1}\left(A_{i}, \mathbb{Z}\right) \oplus H_{1}\left(\widehat{A_{i}}, \mathbb{Z}\right) .
$$

Since $H_{1}\left(\widehat{A_{i}}, \mathbb{Z}\right) \cong H_{1}\left(A_{i}, \mathbb{Z}\right)^{\vee}$, the group $H_{1}\left(A_{i} \times \widehat{A_{i}}, \mathbb{Z}\right)$ is endowed with a natural quadratic form. Indeed, if $\left(a_{1}, \alpha_{1}\right),\left(a_{2}, \alpha_{2}\right) \in H_{1}\left(A_{i} \times \widehat{A_{i}}, \mathbb{Z}\right)$, we define

$$
\left\langle\left(a_{1}, \alpha_{1}\right),\left(a_{2}, \alpha_{2}\right)\right\rangle_{i}:=\alpha_{1}\left(a_{2}\right)+\alpha_{2}\left(a_{1}\right),
$$

where $i \in\{1,2\}$. Consider the set of isomorphisms

$$
\mathrm{U}\left(A_{1}, A_{2}\right):=\left\{f \in \operatorname{Isom}\left(A_{1} \times \widehat{A_{1}}, A_{2} \times \widehat{A_{2}}\right):\left\langle f_{*}\left(a_{1}, \alpha_{1}\right), f_{*}\left(a_{2}, \alpha_{2}\right)\right\rangle_{2}=\left\langle\left(a_{1}, \alpha_{1}\right),\left(a_{2}, \alpha_{2}\right)\right\rangle_{1}\right\} .
$$

Theorem 2.1. ([26], Theorem 2.19 and Proposition 4.12.) Let $A_{1}$ and $A_{2}$ be abelian varieties. If $\Phi_{\mathcal{E}}: \mathrm{D}^{\mathrm{b}}\left(A_{1}\right) \rightarrow \mathrm{D}^{\mathrm{b}}\left(A_{2}\right)$ is an equivalence, then, for any $\mathcal{F} \in \mathrm{D}^{\mathrm{b}}\left(A_{1}\right)$,

$$
F_{\mathcal{E}}(\mathcal{F})=f_{\mathcal{E} *}(\mathcal{F}) \otimes N_{\mathcal{E}}
$$

where $F_{\mathcal{E}}$ is the equivalence in (2.2),$f_{\mathcal{E}} \in \mathrm{U}\left(A_{1}, A_{2}\right)$ and $N_{\mathcal{E}} \in \operatorname{Pic}\left(A_{2} \times \widehat{A_{2}}\right)$. Moreover, there exists a surjective map

$$
\gamma: \operatorname{Eq}\left(\mathrm{D}^{\mathrm{b}}\left(A_{1}\right), \mathrm{D}^{\mathrm{b}}\left(A_{2}\right)\right) \longrightarrow \mathrm{U}\left(A_{1}, A_{2}\right)
$$

such that $\gamma\left(\Phi_{\mathcal{E}}\right)=f_{\mathcal{E}}$, where $\mathrm{Eq}\left(\mathrm{D}^{\mathrm{b}}\left(A_{1}\right), \mathrm{D}^{\mathrm{b}}\left(A_{2}\right)\right)$ is the set of equivalences between $\mathrm{D}^{\mathrm{b}}\left(A_{1}\right)$ and $\mathrm{D}^{\mathrm{b}}\left(A_{2}\right)$.

2.2. Hodge structures and derived categories. If $X$ is a smooth projective variety of dimension $d$, we denote by $\widetilde{H}(X, \mathbb{Q})$ the even cohomology group $H^{2 *}(X, \mathbb{Q})$ with the weight- $d$ Hodge structure defined as follows:

$$
\widetilde{H}^{p, q}(X)=\bigoplus_{p-q=r-s} H^{r, s}(X),
$$

where $H^{r, s}(X)$ is the $(r, s)$-part of the usual Hodge decomposition of $H^{r+s}(X, \mathbb{C}) \subset H^{2 *}(X, \mathbb{C})$. An equivalent way to put on $H^{*}(X, \mathbb{C})$ such a Hodge structure could be obtained considering the natural grading on the Hochschild homology of $X$ (see, for example, [5]).

Suppose now that $X$ is either an abelian or a K3 surface, $H^{2,0}(X)=\left\langle\sigma_{X}\right\rangle$ and $B$ is any class in $H^{2}(X, \mathbb{Q})$. Then

$$
\varphi:=\exp (B)\left(\sigma_{X}\right)=\sigma_{X}+B \wedge \sigma_{X} \in H^{2}(X, \mathbb{C}) \oplus H^{4}(X, \mathbb{C})
$$

is a generalized Calabi-Yau structure on $X$ (for a complete picture see [12]). Let $T(X, B)$ be the minimal primitive sublattice of $H^{2}(X, \mathbb{Z}) \oplus H^{4}(X, \mathbb{Z})$ such that $\varphi \in T(X, B) \otimes \mathbb{C}$. The lattice $T(X, B)$ is the generalized transcendental lattice of $\varphi$ (see [12] and [14]). Let $\widetilde{H}(X, \mathbb{Z})$ be the $\mathbb{Z}$-module $H^{0}(X, \mathbb{Z}) \oplus H^{2}(X, \mathbb{Z}) \oplus H^{4}(X, \mathbb{Z})$ endowed with the Mukai pairing

$$
\left\langle\left(a_{0}, a_{2}, a_{4}\right),\left(b_{0}, b_{2}, b_{4}\right)\right\rangle=a_{2} \cdot b_{2}-a_{0} \cdot b_{4}-a_{4} \cdot b_{0},
$$

where $\left(a_{0}, a_{2}, a_{4}\right),\left(b_{0}, b_{2}, b_{4}\right) \in H^{2 *}(X, \mathbb{Z})$ and "." is the cup-product. We write $\widetilde{H}(X, B, \mathbb{Z})$ for the lattice $\widetilde{H}(X, \mathbb{Z})$ with the weight-two Hodge structure such that

$$
\widetilde{H}^{2,0}(X, B):=\exp (B)\left(\widetilde{H}^{2,0}(X)\right)
$$

and $\widetilde{H}^{1,1}(X, B)$ is its orthogonal complement in $H^{2}(X, \mathbb{C})$. It is clear that $T(X, B)$ inherits from $\widetilde{H}(X, B, \mathbb{Z})$ a weight-two Hodge structure. By definition, $T(X)=T(X, 0)$ is the transcendental lattice of $X$ and

$$
\mathrm{NS}(X):=T(X)^{\perp} \subset H^{2}(X, \mathbb{Z})
$$


is the Néron-Severi group of $X$. The number $\rho(X):=\operatorname{rkNS}(X)$ is the Picard number of $X$. If $L_{1}$ and $L_{2}$ are lattices endowed with a weight- $k$ Hodge structure, then an isometry $f: L_{1} \rightarrow L_{2}$ is a Hodge isometry if it preserves the Hodge structures.

For abelian and K3 surfaces, Orlov proved in [25] (using results in [20]) the following theorem:

Theorem 2.2. ([25], Theorem 3.3.) Let $X_{1}$ and $X_{2}$ be either abelian or K3 surfaces. Then the following two conditions are equivalent:

(i) there exists an equivalence $\mathrm{D}^{\mathrm{b}}\left(X_{1}\right) \cong \mathrm{D}^{\mathrm{b}}\left(X_{2}\right)$;

(ii) there exists a Hodge isometry $T\left(X_{1}\right) \cong T\left(X_{2}\right)$.

\section{Derived Categories of the Smooth Stacks}

This section is mainly devoted to the proof of Theorem 1.1. As it will turn out, such a proof, which will be given in Section 3.2, relies on some results about the equivariant derived categories of coherent sheaves on abelian varieties proved in Section 3.1. In Section 3.3 some geometric applications are discussed.

3.1. Equivariant derived categories and abelian varieties. Consider the simple case of an abelian variety $A$ with the action of $G:=\mathbb{Z} / 2 \mathbb{Z}$ induced by the automorphism $\iota: A \rightarrow A$ such that $\iota(a)=-a$, for any $a \in A$. A $G$-linearization for a coherent sheaf $\mathcal{E} \in \mathbf{C o h}(A)$ is an isomorphism $\lambda: \mathcal{E} \rightarrow \iota^{*} \mathcal{E}$ such that $\iota^{*}(\lambda)=\lambda$ and $\iota^{*}(\lambda) \circ \lambda=\lambda \circ \lambda=\mathrm{id}$.

$\operatorname{Coh}^{G}(A)$ is the abelian category whose objects are the pairs $(\mathcal{E}, \lambda)$, where $\mathcal{E} \in \mathbf{C o h}(A)$ admits a $G$-linearization and $\lambda$ is a $G$-linearization for $\mathcal{E}$. The morphisms in $\mathbf{C o h}^{G}(A)$ are the morphisms in $\operatorname{Coh}(A)$ compatible with the $G$-linearizations. We define $\mathrm{D}_{G}^{\mathrm{b}}(A):=\mathrm{D}^{\mathrm{b}}\left(\mathbf{C o h}^{G}(A)\right)$ to be the bounded derived category of $\mathbf{C} \mathbf{C h}^{G}(A)$. A complete discussion about the general case when $G$ is any finite group acting on a smooth projective variety can be found in [2].

If $A_{1}$ and $A_{2}$ are abelian varieties and $G_{\Delta} \cong \mathbb{Z} / 2 \mathbb{Z}$ acts on $A_{1} \times A_{2}$ via the automorphism $\iota \times \iota$, the set of $G_{\Delta}$-invariant equivalences has the following description:

$$
\mathrm{Eq}\left(\mathrm{D}^{\mathrm{b}}\left(A_{1}\right), \mathrm{D}^{\mathrm{b}}\left(A_{2}\right)\right)^{G_{\Delta}}=\left\{\Phi_{\mathcal{G}} \in \operatorname{Eq}\left(\mathrm{D}^{\mathrm{b}}\left(A_{1}\right), \mathrm{D}^{\mathrm{b}}\left(A_{2}\right)\right): \mathcal{G} \in \mathrm{D}^{\mathrm{b}}\left(A_{1} \times A_{2}\right) \text { is } G_{\Delta} \text {-invariant }\right\} .
$$

An equivalence $\Phi: \mathrm{D}_{G}^{\mathrm{b}}\left(A_{1}\right) \cong \mathrm{D}_{G}^{\mathrm{b}}\left(A_{2}\right)$ is a Fourier-Mukai equivalence if there is an isomorphism as in (2.1), where the kernel $\mathcal{E}$ is in $\mathrm{D}_{G \times G}^{\mathrm{b}}\left(A_{1} \times A_{2}\right)$. $\operatorname{Eq}\left(\mathrm{D}_{G}^{\mathrm{b}}\left(A_{1}\right), \mathrm{D}_{G}^{\mathrm{b}}\left(A_{2}\right)\right)$ is the set whose elements are the equivalences of this type.

Proposition 3.1. Let $A_{1}$ and $A_{2}$ be abelian varieties and let $G=\mathbb{Z} / 2 \mathbb{Z}$ act on $A_{1}$ and $A_{2}$ as above. Then the restriction

$$
\gamma: \operatorname{Eq}\left(\mathrm{D}^{\mathrm{b}}\left(A_{1}\right), \mathrm{D}^{\mathrm{b}}\left(A_{2}\right)\right)^{G_{\Delta}} \longrightarrow \mathrm{U}\left(A_{1}, A_{2}\right)
$$

of the map in (2.3) is surjective and $\mathrm{Eq}\left(\mathrm{D}_{G}^{\mathrm{b}}\left(A_{1}\right), \mathrm{D}_{G}^{\mathrm{b}}\left(A_{2}\right)\right)$ is non-empty if $\mathrm{U}\left(A_{1}, A_{2}\right)$ is non-empty.

Proof. By definition, we can think of any $f \in \mathrm{U}\left(A_{1}, A_{2}\right)$ as represented by a matrix

$$
\left(\begin{array}{cc}
x_{f} & y_{f} \\
z_{f} & w_{f}
\end{array}\right)
$$

Define $S\left(A_{1}, A_{2}\right):=\left\{f \in \mathrm{U}\left(A_{1}, A_{2}\right): y_{f}\right.$ is an isogeny $\}$ and let $f \in S\left(A_{1}, A_{2}\right)$. Using results from [19], Orlov proved in [26] (see, in particular, [26, Prop. 4.12]) that there exists a vector bundle $\mathcal{E}$ on $A_{1} \times A_{2}$ with the following properties:

(a) $\mathcal{E}$ is simple and $\Phi_{\mathcal{E}}$ is an equivalence;

(b) for any $(a, b) \in A_{1} \times A_{2}$, if $T_{(a, b)}$ is the translation with respect to the point $(a, b)$, then $T_{(a, b) *} \mathcal{E} \cong \mathcal{E} \otimes P$ for some $P \in \operatorname{Pic}^{0}\left(A_{1} \times A_{2}\right) ;$

(c) $\gamma\left(\Phi_{\mathcal{E}}\right)=f$. 
Consider now the sheaf $\mathcal{F}:=(\iota \times \iota)^{*} \mathcal{E}$. It is clear that $\gamma\left(\Phi_{\mathcal{F}}\right)=\gamma\left(\Phi_{\mathcal{E}}\right)=f$.

For a brief proof of this fact, consider the maps $\Phi_{i}, \Phi_{\mathcal{P}_{i}}$ and $\mu_{i}$ in (2.2). A straightforward calculation shows that $(\iota \times \iota)^{*} \mathcal{P}_{i} \cong \mathcal{P}_{i}$. Moreover, $\mu_{i}$ is a morphism of abelian varieties. Hence $(\iota \times \iota)^{*} \circ\left(\mathrm{id} \times \Phi_{\mathcal{P}_{i}}\right) \circ(\iota \times \iota)^{*}=\mathrm{id} \times \Phi_{\mathcal{P}_{i}}$ and $(\iota \times \iota)^{*} \circ \mu_{i *} \circ(\iota \times \iota)^{*}=\mu_{i *}$. This implies that $(\iota \times \iota)^{*} \circ \Phi_{i} \circ(\iota \times \iota)^{*}=\Phi_{i}$, for $i \in\{1,2\}$. Since $\Phi_{\mathcal{F}}=\iota^{*} \circ \Phi_{\mathcal{E}} \circ \iota^{*}$ and $\Phi_{\mathcal{F}} \times \Phi_{\mathcal{F}_{R}}=(\iota \times \iota)^{*} \circ\left(\Phi_{\mathcal{E}} \times\right.$ $\left.\Phi_{\mathcal{E}_{R}}\right) \circ(\iota \times \iota)^{*}$, we rewrite the commutative diagram (2.2) in the following way:

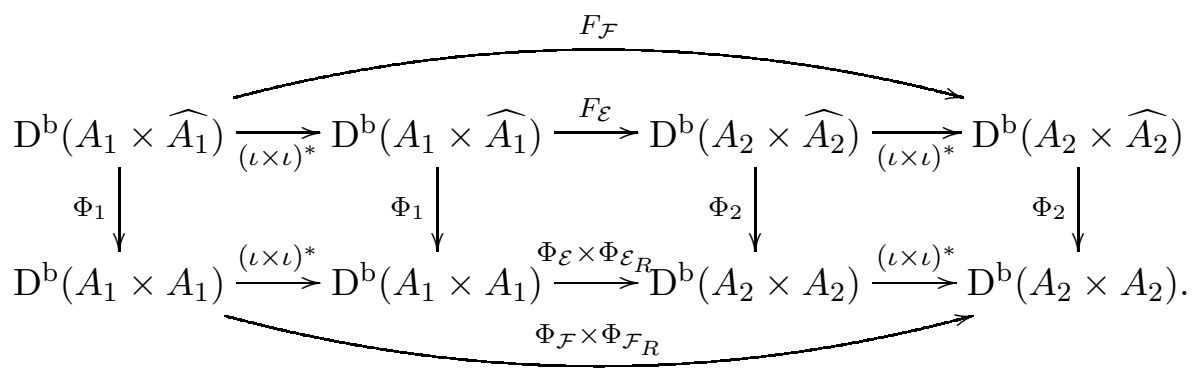

By Theorem 2.1, for any $\mathcal{G} \in \mathrm{D}^{\mathrm{b}}\left(A_{1} \times \widehat{A_{1}}\right), F_{\mathcal{F}}(\mathcal{G})=f_{\mathcal{F} *}(\mathcal{G}) \otimes N_{\mathcal{F}}$, for some $f_{\mathcal{F}} \in \mathrm{U}\left(A_{1}, A_{2}\right)$ and $N_{\mathcal{F}} \in \operatorname{Pic}\left(A_{2} \times \widehat{A_{2}}\right)$. Hence, from (3.1) we deduce that

$$
\begin{aligned}
F_{\mathcal{F}}(\mathcal{G}) & =\left((\iota \times \iota)^{*} \circ F_{\mathcal{E}} \circ(\iota \times \iota)^{*}\right)(\mathcal{G}) \\
& =\left((\iota \times \iota)^{*} \circ f_{\mathcal{E} *} \circ(\iota \times \iota)^{*}\right)(\mathcal{G}) \otimes M \\
& =f_{\mathcal{E} *}(\mathcal{G}) \otimes M,
\end{aligned}
$$

for some $M \in \operatorname{Pic}\left(A_{2} \times \widehat{A_{2}}\right)$. Observe that the last equality holds true because $f_{\mathcal{E}}$ is a morphism of abelian varieties. This proves that $\gamma\left(\Phi_{\mathcal{F}}\right)=\gamma\left(\iota^{*} \circ \Phi_{\mathcal{E}} \circ \iota^{*}\right)=\gamma\left(\Phi_{\mathcal{E}}\right)$ which is what we claimed.

Due to this last remark and to [26, Cor. 3.4], there exist $a \in A_{1}$ and $\alpha \in \widehat{A_{1}}$ such that

$$
\mathcal{F}=T_{(a, 0) *} \mathcal{E} \otimes p^{*} P_{\alpha}[i],
$$

where $p: A_{1} \times A_{2} \rightarrow A_{1}$ is the projection, $i$ is an integer and $P_{\alpha}$ is the degree zero line bundle on $A_{1}$ corresponding to $\alpha$. In the following arguments, without loss of generality, we will forget about the shift $[i]$ in (3.2).

Since $\mathcal{E}$ satisfies (b), from (3.2) we get $\mathcal{F}=\mathcal{E} \otimes Q$, where $Q$ is a degree zero line bundle on $A_{1} \times A_{2}$. Let $N \in \operatorname{Pic}^{0}\left(A_{1} \times A_{2}\right)$ be such that $N^{2}=Q$ and consider the sheaf $\mathcal{E}_{f}:=\mathcal{E} \otimes N$. It is easy to see that

$$
\begin{aligned}
(\iota \times \iota)^{*}\left(\mathcal{E}_{f}\right) & =(\iota \times \iota)^{*}(\mathcal{E} \otimes N) \\
& \cong \mathcal{E} \otimes Q \otimes N^{\vee} \\
& \cong \mathcal{E} \otimes N \\
& =\mathcal{E}_{f} .
\end{aligned}
$$

Due to [26, Prop. 3.3] and to (c), $\gamma\left(\Phi_{\mathcal{E}_{f}}\right)=\gamma\left(\Phi_{\mathcal{E}}\right)=f$.

Let $f \in \mathrm{U}\left(A_{1}, A_{2}\right)$. Orlov observed in [26, Sect. 4] that there exist $g_{1} \in S\left(A_{1}, A_{2}\right)$ and $g_{2} \in$ $S\left(A_{2}, A_{2}\right)$ such that $f=g_{2} \circ g_{1}$. From its very definition, the map $\gamma$ in Theorem 2.1 preserves the compositions. Hence $\gamma$ restricts to a surjective map $\gamma: \operatorname{Eq}\left(\mathrm{D}^{\mathrm{b}}\left(A_{1}\right), \mathrm{D}^{\mathrm{b}}\left(A_{2}\right)\right)^{G_{\Delta}} \rightarrow \mathrm{U}\left(A_{1}, A_{2}\right)$.

To prove the second claim in Proposition 3.1, consider the set

$$
\operatorname{Ker}\left(A_{1}, A_{2}, G_{\Delta}\right):=\left\{(\mathcal{G}, \lambda) \in \mathrm{D}_{G_{\Delta}}^{\mathrm{b}}\left(A_{1} \times A_{2}\right): \Phi_{\mathcal{G}} \in \operatorname{Eq}\left(\mathrm{D}^{\mathrm{b}}\left(A_{1}\right), \mathrm{D}^{\mathrm{b}}\left(A_{2}\right)\right)\right\} .
$$

Since the group cohomology $H^{2}\left(\mathbb{Z} / 2 \mathbb{Z}, \mathbb{C}^{*}\right)$ is trivial, [27, Thm. 6] shows the existence of two maps

$$
\begin{aligned}
& \psi_{1}: \operatorname{Ker}\left(A_{1}, A_{2}, G_{\Delta}\right) \longrightarrow \operatorname{Eq}\left(\mathrm{D}^{\mathrm{b}}\left(A_{1}\right), \mathrm{D}^{\mathrm{b}}\left(A_{2}\right)\right)^{G_{\Delta}} \\
& \psi_{2}: \operatorname{Ker}\left(A_{1}, A_{2}, G_{\Delta}\right) \longrightarrow \operatorname{Eq}\left(\mathrm{D}_{G}^{\mathrm{b}}\left(A_{1}\right), \mathrm{D}_{G}^{\mathrm{b}}\left(A_{2}\right)\right)
\end{aligned}
$$

such that, for any $(\mathcal{G}, \lambda) \in \operatorname{Ker}\left(A_{1}, A_{2}, G_{\Delta}\right), \psi_{1}((\mathcal{G}, \lambda))=\Phi_{\mathcal{G}}$ and $\psi_{2}((\mathcal{G}, \lambda))=\Phi_{\mathcal{H}}$, where $\mathcal{H}:=$ $\left(\mathcal{G} \oplus(\iota, \text { id })^{*} \mathcal{G}, \lambda^{\prime}\right)$ and $\lambda^{\prime}$ is the natural $(G \times G)$-linearization induced by $\lambda$. 
We previously proved that for any $f \in S\left(A_{1}, A_{2}\right)$, there exists $\Phi_{\mathcal{E}_{f}} \in \operatorname{Eq}\left(\mathrm{D}^{\mathrm{b}}\left(A_{1}\right), \mathrm{D}^{\mathrm{b}}\left(A_{2}\right)\right)^{G_{\Delta}}$ such that $\gamma\left(\Phi_{\mathcal{E}_{f}}\right)=f$. From [27] it follows that $\psi_{1}$ is surjective and that the $\operatorname{set} \operatorname{Ker}\left(A_{1}, A_{2}, G_{\Delta}\right)$ is non-empty if $\operatorname{Eq}\left(\mathrm{D}^{\mathrm{b}}\left(A_{1}\right), \mathrm{D}^{\mathrm{b}}\left(A_{2}\right)\right)^{G_{\Delta}}$ is non-empty. Hence, there exists $\Psi_{f} \in \operatorname{Ker}\left(A_{1}, A_{2}, G_{\Delta}\right)$ such that $\psi_{1}\left(\Psi_{f}\right)=\Phi_{\mathcal{E}_{f}}$. The functor $\psi_{2}\left(\Psi_{f}\right)$ is in $\operatorname{Eq}\left(\mathrm{D}_{G}^{\mathrm{b}}\left(A_{1}\right), \mathrm{D}_{G}^{\mathrm{b}}\left(A_{2}\right)\right)$.

The special case $A_{1}=A_{2}$ is also treated in [27].

3.2. Proof of Theorem 1.1. Let $A_{1}$ and $A_{2}$ be abelian varieties and suppose that $\mathrm{D}^{\mathrm{b}}\left(A_{1}\right) \cong$ $\mathrm{D}^{\mathrm{b}}\left(A_{2}\right)$. Due to Theorem 2.1, the set $\mathrm{U}\left(A_{1}, A_{2}\right)$ is non-empty. Therefore, if $G=\mathbb{Z} / 2 \mathbb{Z}$ acts on $A_{1}$ and $A_{2}$ as prescribed at the beginning of Section 3.1, then Proposition 3.1 yields an equivalence $\Psi: \mathrm{D}_{G}^{\mathrm{b}}\left(A_{1}\right) \stackrel{\sim}{\rightarrow} \mathrm{D}_{G}^{\mathrm{b}}\left(A_{2}\right)$.

Consider the stacks $\left[A_{1} / G\right]$ and $\left[A_{2} / G\right]$ (see [9] and [16]). For any $i \in\{1,2\}$, let $\mathrm{D}^{\mathrm{b}}\left(\left[A_{i} / G\right]\right.$ ) be the bounded derived category of the abelian category $\mathbf{C o h}\left(\left[A_{i} / G\right]\right)$ of coherent sheaves on $\left[A_{i} / G\right]$ (see [16]). Obviously $\mathrm{D}^{\mathrm{b}}\left(\left[A_{i} / G\right]\right) \cong \mathrm{D}_{G}^{\mathrm{b}}\left(A_{i}\right)$, because $\mathbf{C o h}\left(\left[A_{i} / G\right]\right) \cong \mathbf{C o h}^{G}\left(A_{i}\right)$, for any $i \in\{1,2\}$. This implies that $\Psi$ can be rewritten as $\Phi: \mathrm{D}^{\mathrm{b}}\left(\left[A_{1} / G\right]\right) \stackrel{\sim}{\rightarrow} \mathrm{D}^{\mathrm{b}}\left(\left[A_{2} / G\right]\right)$. Due to [16], $\Phi$ is of Fourier-Mukai type (i.e. there is an isomorphism as in (2.1) $)$. Hence, the first part of Theorem 1.1 is proved.

Assume that an equivalence $\Phi: \mathrm{D}^{\mathrm{b}}\left(\left[A_{1} / G\right]\right) \stackrel{\sim}{\rightarrow} \mathrm{D}^{\mathrm{b}}\left(\left[A_{2} / G\right]\right)$ is given. As before, the results in [16 imply that we can think of $\Phi$ as a Fourier-Mukai equivalence whose kernel is a $(G \times G)$-linearized complex $(\mathcal{E}, \lambda)$. Obviously, the inverse $\Phi^{-1}$ is a Fourier-Mukai equivalence as well. Suppose that its kernel is $\left(\mathcal{F}, \lambda^{\prime}\right)$. It is an easy exercise to show that the kernel of the identity functor id $=$ $\Phi \circ \Phi^{-1}: \mathrm{D}_{G}^{\mathrm{b}}\left(A_{i}\right) \rightarrow \mathrm{D}_{G}^{\mathrm{b}}\left(A_{i}\right)$ is the $(G \times G)$-linearized sheaf $\left(\mathcal{O}_{\Delta} \oplus(\iota, \mathrm{id})^{*} \mathcal{O}_{\Delta}, \mu\right)$, where $\mu$ is the natural linearization and $\Delta \hookrightarrow A_{i} \times A_{i}$ is the diagonal embedding.

Consider the functors $\Phi_{\mathcal{E}}, \Phi_{\mathcal{F}}$ and $\Phi_{\mathcal{O}_{\Delta} \oplus(\iota, \mathrm{id})^{*} \mathcal{O}_{\Delta}}$. Although they are no longer equivalences, they induce the commutative diagram

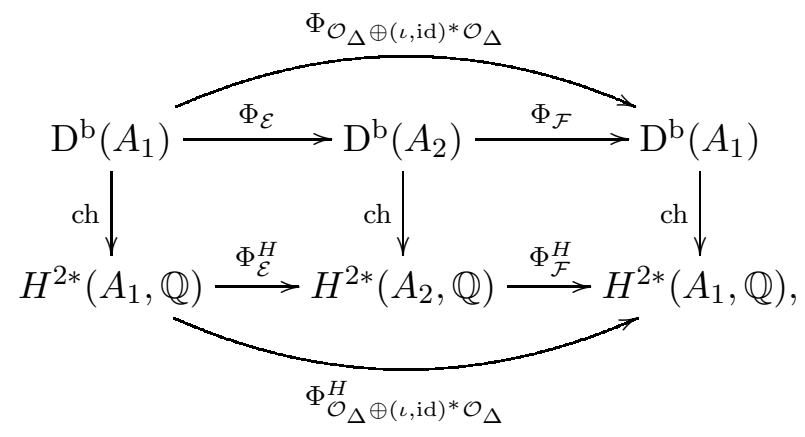

where $\Phi_{\mathcal{E}}^{H}: H^{2 *}\left(A_{1}, \mathbb{Q}\right) \rightarrow H^{2 *}\left(A_{2}, \mathbb{Q}\right)$ is such that $\Phi_{\mathcal{E}}^{H}(a)=p_{2 *}\left(\operatorname{ch}(\mathcal{E}) \cdot p_{1}^{*}(a)\right)$ and $p_{i}: A_{1} \times A_{2} \rightarrow A_{i}$ is the projection. Take analogous definitions for $\Phi_{\mathcal{F}}^{H}$ and $\Phi_{\mathcal{O}_{\Delta} \oplus(\iota, \text { id })^{*} \mathcal{O}_{\Delta}}^{H}$.

Observe that $(\iota, \mathrm{id})^{*} \mathcal{O}_{\Delta}$ is the kernel of the Fourier-Mukai equivalence $\iota^{*}: \mathrm{D}^{\mathrm{b}}\left(A_{i}\right) \stackrel{\sim}{\rightarrow} \mathrm{D}^{\mathrm{b}}\left(A_{i}\right)$. Since $\iota^{*}$ acts as the identity on the cohomology lattice $\widetilde{H}\left(A_{i}, \mathbb{Z}\right)$, from (3.3) we deduce

$$
\Phi_{\mathcal{F}}^{H} \circ \Phi_{\mathcal{E}}^{H}=\left(\Phi_{\mathcal{O}_{\Delta} \oplus(\iota, \mathrm{id}) * \mathcal{O}_{\Delta}}\right)^{H}=2 \mathrm{id} .
$$

Hence $\Phi_{\mathcal{E}}^{H}$ is injective. Exchanging the roles of $\Phi_{\mathcal{E}}$ and $\Phi_{\mathcal{F}}$ in (3.3), we see that $\Phi_{\mathcal{E}}^{H}$ is an isomorphism of $\mathbb{Q}$-vector spaces. In particular, $\operatorname{dim}\left(A_{1}\right)=\operatorname{dim}\left(A_{2}\right)=n$.

The fact that the Hodge structures defined in (2.4) are preserved follows from the standard argument for Fourier-Mukai equivalences (see [13, Prop. 5.39]). Indeed, one just needs to observe that $\operatorname{ch}(\mathcal{E}) \in \widetilde{H}^{2 n, 2 n}\left(A_{1} \times A_{2}\right)$. This concludes the proof of Theorem 1.1.

Remark 3.2. Of course, in general, $\Phi_{\mathcal{E}}^{H}$ does not preserve the Mukai pairing naturally defined on $H^{2 *}\left(A_{i}, \mathbb{Q}\right)$ by means of the cup product $([13$, Chapter 5$])$. Indeed, it is easy to see that the Mukai pairing is preserved up to a factor 2 . 
3.3. Geometric applications. Assume that $A_{1}$ and $A_{2}$ are abelian surfaces. The main result in [2] yields an equivalence $\Psi_{i}: \mathrm{D}^{\mathrm{b}}\left(\left[A_{i} /\langle\iota\rangle\right]\right) \stackrel{\sim}{\rightarrow} \mathrm{D}^{\mathrm{b}}\left(\operatorname{Km}\left(A_{i}\right)\right)$, for any $i \in\{1,2\}$. Thus, if $\Upsilon$ : $\mathrm{D}^{\mathrm{b}}\left(A_{1}\right) \stackrel{\sim}{\rightarrow} \mathrm{D}^{\mathrm{b}}\left(A_{2}\right)$ is a Fourier-Mukai equivalence, we immediately get a second Fourier-Mukai equivalence

$$
\Phi: \mathrm{D}^{\mathrm{b}}\left(\operatorname{Km}\left(A_{1}\right)\right) \stackrel{\Psi_{1}^{-1}}{\longrightarrow} \mathrm{D}^{\mathrm{b}}\left(\left[A_{1} /\langle\iota\rangle\right]\right) \stackrel{\sim}{\rightarrow} \mathrm{D}^{\mathrm{b}}\left(\left[A_{2} /\langle\iota\rangle\right]\right) \stackrel{\Psi_{2}}{\longrightarrow} \mathrm{D}^{\mathrm{b}}\left(\operatorname{Km}\left(A_{2}\right)\right),
$$

where the middle equivalence is produced by Theorem 1.1 and the kernel of $\Phi$ can be easily computed using [2]. This leads to a different and explicit proof of the "only if" implication in (A) without using the lattice theoretical description of the transcendental lattices of an abelian surface and of the associated Kummer surface.

Let us discuss a second geometric application. Assume that $A$ is an abelian surface. We denote by $\mathrm{K}^{n}(A)$ the $n$-th generalized Kummer variety of $A$. Recalling the construction in [1], we see that $\mathrm{K}^{n}(A)$ is the fiber over 0 with respect to the map $\Psi$ which is the composition of the morphisms in the following diagram:

$$
\Psi: \operatorname{Hilb}^{n+1}(A) \stackrel{\rho}{\longrightarrow} \operatorname{Sym}^{n+1}(A) \stackrel{\sigma}{\longrightarrow} A,
$$

where $\rho$ is the Hilbert-Chow morphism and $\sigma\left(a_{1}, \ldots, a_{n+1}\right)=a_{1}+\ldots+a_{n+1}$. It is easy to see that $\mathrm{K}^{n}(A)$ is smooth and that $\mathrm{K}^{1}(A)=\mathrm{Km}(A)$. Furthermore, in [1] Beauville proved that these varieties are examples of irreducible symplectic manifolds.

Proposition 3.3. Let $A$ be an abelian surface and let $n \geq 2$ be an integer. The number of generalized Kummer varieties $\mathrm{K}^{n}(B)$ birational to $\mathrm{K}^{n}(A)$ is finite up to isomorphisms. Moreover if $\mathrm{K}^{n}(A)$ and $\mathrm{K}^{n}(B)$ are birational, then $\mathrm{Km}(A) \cong \mathrm{Km}(B)$ and $A$ and $B$ are isogenous.

Proof. Let $A_{1}$ and $A_{2}$ be abelian surfaces and let $\varphi$ be a birational morphism between $\mathrm{K}^{n}\left(A_{1}\right)$ and $\mathrm{K}^{n}\left(A_{2}\right)$. Obviously, $\varphi$ induces an isomorphism $g: H^{2}\left(\mathrm{~K}^{n}\left(A_{1}\right), \mathbb{Z}\right) \stackrel{\sim}{\rightarrow} H^{2}\left(\mathrm{~K}^{n}\left(A_{2}\right), \mathbb{Z}\right)$. Furthermore, there exists an isometry of lattices $H^{2}\left(\mathrm{~K}^{n}\left(A_{i}\right), \mathbb{Z}\right) \stackrel{\sim}{\rightarrow} H^{2}\left(A_{i}, \mathbb{Z}\right) \oplus \mathbb{Z}\left[E_{i}\right]$, where $E_{i}$ is the restriction to $\mathrm{K}^{n}(A)$ of the exceptional locus of $\operatorname{Hilb}^{n+1}\left(A_{i}\right)$. The left hand side of the isomorphism is endowed with the Beauville-Bogomolov form while the quadratic form on $H^{2}\left(A_{i}, \mathbb{Z}\right)$ is the cup-product (see [1, Lemma 4.10] and [30, Prop. 4.11]).

Since $E_{1}$ and $E_{2}$ are algebraic, $g$ yields an isomorphism $T\left(A_{1}\right) \cong T\left(A_{2}\right)$. Using Theorem 2.2, we get an equivalence $\mathrm{D}^{\mathrm{b}}\left(A_{1}\right) \cong \mathrm{D}^{\mathrm{b}}\left(A_{2}\right)$. To prove that $A_{1}$ is isogenous to $A_{2}$ observe that if $\mathrm{D}^{\mathrm{b}}\left(A_{1}\right) \cong \mathrm{D}^{\mathrm{b}}\left(A_{2}\right)$, then $A_{1} \times \widehat{A_{1}} \cong A_{2} \times \widehat{A_{2}}$ (Theorem 2.1). Hence $A_{1} \times A_{1}$ and $A_{2} \times A_{2}$ are isogenous and $A_{1}$ and $A_{2}$ are isogenous as well. On the other hand, as there are only finitely many isomorphism classes of abelian surfaces $A$ such that $\mathrm{D}^{\mathrm{b}}(A) \cong \mathrm{D}^{\mathrm{b}}\left(A_{1}\right)$ (see [3, Prop. 5.3]), the number of generalized Kummer varieties $\mathrm{K}^{n}\left(A_{2}\right)$ birational to $\mathrm{K}^{n}\left(A_{1}\right)$ is finite up to isomorphism. Moreover, Theorem 1.1 yields an equivalence $\mathrm{D}^{\mathrm{b}}\left(\left[A_{1} /\langle\iota\rangle\right]\right) \cong \mathrm{D}^{\mathrm{b}}\left(\left[A_{2} /\langle\iota\rangle\right]\right)$. Due to (3.4) and Theorem 1.1, $\mathrm{D}^{\mathrm{b}}(\mathrm{Km}(A)) \cong \mathrm{D}^{\mathrm{b}}(\mathrm{Km}(B))$ and then $\operatorname{Km}(A) \cong \mathrm{Km}(B)$ (see [20]).

An analogous result for Hilbert schemes of points on K3 surfaces was proved in [27].

Remark 3.4. Observe that, in general, if $A$ and $B$ are abelian surfaces such that $\operatorname{Km}(A) \cong \operatorname{Km}(B)$, then $\mathrm{K}^{n}(A)$ and $\mathrm{K}^{n}(B)$ are not necessarily birational. Indeed, consider an abelian surface $A$ such that $A \neq \widehat{A}$ and $\operatorname{NS}(A)=\langle H\rangle$ with $H^{2}=6$. Obviously $\mathrm{D}^{\mathrm{b}}(A) \cong \mathrm{D}^{\mathrm{b}}(\widehat{A})$. Due to Theorem 1.1, $\mathrm{D}^{\mathrm{b}}(\operatorname{Km}(A)) \cong \mathrm{D}^{\mathrm{b}}(\operatorname{Km}(\widehat{A}))$ and $\operatorname{Km}(A) \cong \operatorname{Km}(\widehat{A})([20])$. On the other hand, Namikawa ([21, Sect. 5]) proved that $\mathrm{K}^{2}(A)$ and $\mathrm{K}^{2}(\widehat{A})$ are not birational.

Furthermore, Example 4.3 yields very explicit examples of isogenous abelian surfaces $A$ and $B$ such that $\mathrm{D}^{\mathrm{b}}(A) \neq \mathrm{D}^{\mathrm{b}}(B)$. In particular $\mathrm{Km}(A) \neq \mathrm{Km}(B)$ and $\mathrm{K}^{n}(A)$ is not birational to $\mathrm{K}^{n}(B)$ for any positive integer $n$. 


\section{Derived CATEgories of TWisted Kummer surfaces}

In this section we prove Theorem 1.2 which relates the existence of equivalences between the twisted derived categories of two Kummer surfaces and the existence of Hodge isometries between the generalized transcendental lattices of the corresponding abelian surfaces. More precisely, in Section 4.1 we introduce the Brauer group of smooth projective varieties and we prove a preliminary result (Lemma 4.1) which will be used in the proof of Theorem 1.2 contained in Section 4.2. We also discuss a geometric example and an application to the problem of determining the number of possible twisted Kummer structures on a twisted K3 surface (respectively in Sections 4.3 and 4.4).

4.1. Brauer groups and twisted sheaves. Recall that the Brauer group $\operatorname{Br}(X)$ of a smooth projective variety $X$ is the torsion part of $H^{2}\left(X, \mathcal{O}_{X}^{*}\right)$ in the analytic topology (or, equivalently, $H_{\text {ét }}^{2}\left(X, \mathcal{O}_{X}^{*}\right)$ in the étale topology) (see [4, 7]).

Assume that $X$ is either a K3 or an abelian surface. It is known that any $\alpha \in \operatorname{Br}(X)$ is determined (not uniquely) by some $B \in H^{2}(X, \mathbb{Q}$ ) (see Chapter 1 of 4 for the case of K3 surfaces and use a similar argument to deal with abelian surfaces). This follows from the fact that $H^{2}(X, \mathbb{Z})$ is unimodular and $H_{1}(X, \mathbb{Z})$ is torsion free. More precisely, we deduce the existence of natural isomorphisms $\operatorname{Br}(X) \cong T(X)^{\vee} \otimes \mathbb{Q} / \mathbb{Z} \cong \operatorname{Hom}(T(X), \mathbb{Q} / \mathbb{Z})$ and for any $t \in T(X), \alpha: t \longmapsto t \cdot B$ $(\bmod \mathbb{Z})$, where "." is the cup-product. From this we get a surjective map

$$
\kappa_{X}: H^{2}(X, \mathbb{Q}) \longrightarrow \operatorname{Br}(X) .
$$

Lemma 4.1. If $A$ is an abelian surface, there exists an isomorphism $\Theta_{A}: \operatorname{Br}(A) \rightarrow \operatorname{Br}(\operatorname{Km}(A))$.

Proof. The $\mathrm{K} 3$ surface $\mathrm{Km}(A)$ is the crepant resolution of $\mathrm{K}(A)=A /\langle\iota\rangle$. Hence there exists a rational map $\pi: A \rightarrow \operatorname{Km}(A)$. Furthermore, as it was observed in Remark 2 of [22] (see also [17, Sect. 4]), the homomorphism $\pi_{*}$ induces a Hodge isometry

$$
\pi_{*}: T(A)(2) \longrightarrow T(\mathrm{Km}(A)) \text {. }
$$

(Recall that, given a lattice $L$ with quadratic form $b_{L}$, the lattice $L(m)$, with $m \in \mathbb{Z}$, coincides with $L$ as a group but its quadratic form $b_{L(m)}$ is such that $b_{L(m)}\left(l_{1}, l_{2}\right)=m b_{L}\left(l_{1}, l_{2}\right)$, for any $l_{1}, l_{2} \in L$.)

In particular, we get a natural morphism $\Xi: H^{2}(A, \mathbb{Q}) \rightarrow T(\operatorname{Km}(A)) \otimes \mathbb{Q}$ defined by

$$
\Xi: B \longmapsto \frac{\pi_{*}(p(B))}{2},
$$

where $p: H^{2}(A, \mathbb{Q}) \rightarrow T(A) \otimes \mathbb{Q}$ is the orthogonal projection. This yields a morphism $\Theta_{A}:$ $\mathrm{Br}(A) \longrightarrow \operatorname{Br}(\mathrm{Km}(A))$ of Brauer groups defined by the commutative diagram

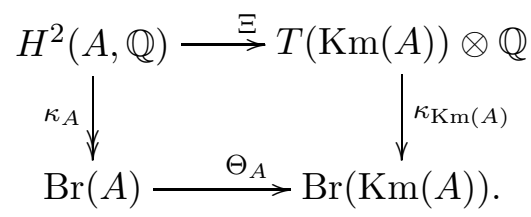

Observe that $\Theta_{A}$ is well-defined because, obviously, the restriction $\left.\kappa_{\operatorname{Km}(A)}\right|_{T(\operatorname{Km}(A)) \otimes \mathbb{Q}}$ is still surjective. An easy check then shows that $\Theta_{A}$ is an isomorphism.

Any $\alpha \in \operatorname{Br}(X)$ can be represented by a Čech 2-cocycle on an analytic cover $\left\{U_{i}\right\}_{i \in I}$ of $X$ using sections $\alpha_{i j k} \in \Gamma\left(U_{i} \cap U_{j} \cap U_{k}, \mathcal{O}_{X}^{*}\right)$. An $\alpha$-twisted coherent sheaf $\mathcal{F}$ is a pair $\left(\left\{\mathcal{F}_{i}\right\}_{i \in I},\left\{\varphi_{i j}\right\}_{i, j \in I}\right)$, where $\mathcal{F}_{i}$ is a coherent sheaf on $U_{i}$ and $\varphi_{i j}:\left.\left.\mathcal{F}_{j}\right|_{U_{i} \cap U_{j}} \rightarrow \mathcal{F}_{i}\right|_{U_{i} \cap U_{j}}$ is an isomorphism such that $\varphi_{i i}=\mathrm{id}, \varphi_{j i}=\varphi_{i j}^{-1}$ and $\varphi_{i j} \circ \varphi_{j k} \circ \varphi_{k i}=\alpha_{i j k}$ id. Given $\alpha \in \operatorname{Br}(X)$, we denote by $\operatorname{Coh}(X, \alpha)$ the abelian category of $\alpha$-twisted coherent sheaves on $X$ while $\mathrm{D}^{\mathrm{b}}(X, \alpha):=\mathrm{D}^{\mathrm{b}}(\mathbf{C o h}(X, \alpha))$ is the bounded derived category of $\operatorname{Coh}(X, \alpha)$ (see [4] and 15] for details). 
If $X$ and $Y$ are smooth projective varieties and $\alpha \in \operatorname{Br}(X)$ while $\beta \in \operatorname{Br}(Y)$, an equivalence $\Phi: \mathrm{D}^{\mathrm{b}}(X, \alpha) \rightarrow \mathrm{D}^{\mathrm{b}}(Y, \beta)$ is a twisted Fourier-Mukai equivalence if and only if it there is an isomorphism as in (2.1) whose kernel $\mathcal{E}$ is in $\mathrm{D}^{\mathrm{b}}\left(X \times Y, \alpha^{-1} \otimes \beta\right)$ (see also [6]).

As in [14], a twisted variety is a pair $(X, \alpha)$, where $X$ is a smooth projective variety and $\alpha \in$ $\operatorname{Br}(X)$. An isomorphism $f:(X, \alpha) \rightarrow(Y, \beta)$ of the twisted varieties $(X, \alpha)$ and $(Y, \beta)$ is an isomorphism $f: X \rightarrow Y$ such that $f^{*} \beta=\alpha$.

4.2. Proof of Theorem 1.2. First of all, observe that, if $X$ is either a K3 or an abelian surface and $\alpha \in \operatorname{Br}(X)$, the lattice $T(X, \alpha):=\operatorname{ker}(\alpha)$ inherits from $T(X)$ a weight-two Hodge structure. Secondly, if $\Theta_{A_{i}}: \operatorname{Br}\left(A_{i}\right) \rightarrow \operatorname{Br}\left(\operatorname{Km}\left(A_{i}\right)\right)$ is the isomorphism in Lemma 4.1, the isometry $\pi_{i *}$ : $T\left(A_{i}\right)(2) \rightarrow T\left(\mathrm{Km}\left(A_{i}\right)\right)$ defined in (4.1) yields a Hodge isometry

$$
f_{i}: T\left(A_{i}, \alpha\right)(2) \longrightarrow T\left(\operatorname{Km}\left(A_{i}\right), \Theta_{A_{i}}(\alpha)\right),
$$

for any $\alpha \in \operatorname{Br}\left(A_{i}\right)$ and $i \in\{1,2\}$.

Proposition 4.7 in [12], originally proved for $\mathrm{K} 3$ surfaces, works perfectly in the case of abelian surfaces as well. Therefore if $X$ is either a $\mathrm{K} 3$ or an abelian surface, $\alpha \in \operatorname{Br}(X)$ and $B \in H^{2}(X, \mathbb{Q})$ is such that $\alpha=\kappa_{X}(B)$, then there exists a Hodge isometry

$$
\begin{aligned}
\exp (B): T(X, \alpha)(k) & \longrightarrow T(X, B)(k) \\
\gamma & \longmapsto(\gamma, B \wedge \gamma),
\end{aligned}
$$

for any $k \in\{1,2\}$. Given $B_{i} \in H^{2}\left(A_{i}, \mathbb{Q}\right)$, let $\widetilde{B}_{i} \in H^{2}\left(\operatorname{Km}\left(A_{i}\right), \mathbb{Q}\right)$ be such that

$$
\Theta_{A_{i}}\left(\kappa_{A_{i}}\left(B_{i}\right)\right)=\kappa_{\mathrm{Km}\left(A_{i}\right)}\left(\widetilde{B}_{i}\right) \text {. }
$$

Define $\widetilde{\alpha}_{i}:=\Theta_{A_{i}}\left(\kappa_{A_{i}}\left(B_{i}\right)\right)$. If $g: T\left(A_{1}, B_{1}\right) \rightarrow T\left(A_{2}, B_{2}\right)$ is a Hodge isometry, the diagram

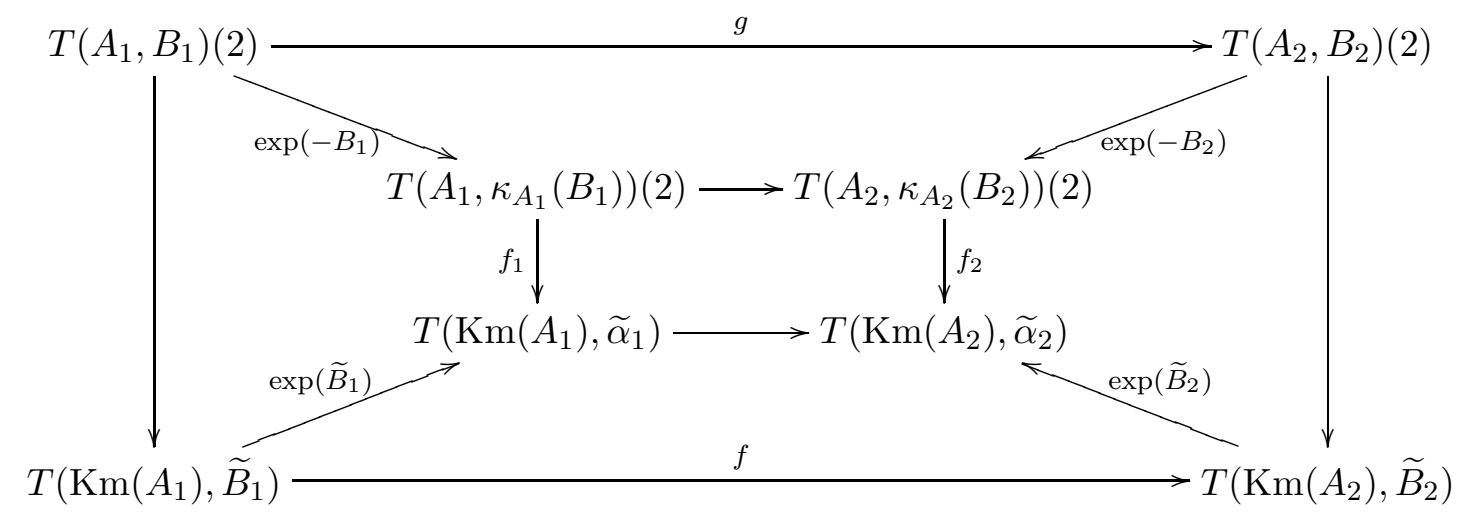

commutes and yields a Hodge isometry $f: T\left(\operatorname{Km}\left(A_{1}\right), \widetilde{B}_{1}\right) \rightarrow T\left(\operatorname{Km}\left(A_{2}\right), \widetilde{B}_{2}\right)$. Conversely, since $\Theta_{i}$ is an isomorphism (Lemma 4.1), the same diagram and remarks show that any Hodge isometry between the generalized transcendental lattices of $\operatorname{Km}\left(A_{1}\right)$ and $\operatorname{Km}\left(A_{2}\right)$ determined by some $\widetilde{B}_{i} \in$ $H^{2}\left(\mathrm{Km}\left(A_{i}\right), \mathbb{Q}\right)$ induces a Hodge isometry of the generalized transcendental lattices of $A_{1}$ and $A_{2}$ corresponding to $B_{i} \in H^{2}\left(A_{i}, \mathbb{Q}\right)$ such that

$$
\kappa_{A_{i}}\left(B_{i}\right)=\Theta_{A_{i}}^{-1}\left(\kappa_{\operatorname{Km}\left(A_{i}\right)}\left(\widetilde{B}_{i}\right)\right) \in \operatorname{Br}\left(A_{i}\right) .
$$

Since the Picard number of $\operatorname{Km}\left(A_{i}\right)$ is greater than 11, the equivalence between item (i) and item (ii) of Theorem 1.2 follows from [14, Thm. 0.4]. Indeed such a result proves that, for any $B_{i} \in H^{2}\left(\mathrm{Km}\left(A_{i}\right), \mathbb{Q}\right)$, there exists a twisted Fourier-Mukai equivalence

$$
\mathrm{D}^{\mathrm{b}}\left(\operatorname{Km}\left(A_{1}\right), \kappa_{\operatorname{Km}\left(A_{1}\right)}\left(B_{1}\right)\right) \cong \mathrm{D}^{\mathrm{b}}\left(\operatorname{Km}\left(A_{2}\right), \kappa_{\mathrm{Km}\left(A_{2}\right)}\left(B_{2}\right)\right)
$$

if and only if there exists a Hodge isometry $T\left(\mathrm{Km}\left(A_{1}\right), B_{1}\right) \cong T\left(\mathrm{Km}\left(A_{2}\right), B_{2}\right)$.

Due to what we have just proved, any twisted Fourier-Mukai equivalence $\mathrm{D}^{\mathrm{b}}\left(\operatorname{Km}\left(A_{1}\right), \alpha_{1}\right) \cong$ $\mathrm{D}^{\mathrm{b}}\left(\operatorname{Km}\left(A_{2}\right), \alpha_{2}\right)$ induces a Hodge isometry $T\left(\operatorname{Km}\left(A_{1}\right)\right) \otimes \mathbb{Q} \cong T\left(\operatorname{Km}\left(A_{2}\right)\right) \otimes \mathbb{Q}$ which yields a 
Hodge isometry $T\left(A_{1}\right) \otimes \mathbb{Q} \cong T\left(A_{2}\right) \otimes \mathbb{Q}$. Consider the Kuga-Satake varieties $\operatorname{KS}\left(A_{1}\right)$ and $\operatorname{KS}\left(A_{2}\right)$ associated to the weight-two Hodge structures on $T\left(A_{1}\right) \otimes \mathbb{Q}$ and $T\left(A_{2}\right) \otimes \mathbb{Q}$ (see Section 4 in [18] for the definition). Theorem 4.3 and Lemma 4.4 in [18] show that, for any $i \in\{1,2\}$,

$$
\underbrace{\operatorname{KS}\left(A_{i}\right) \times \cdots \times \operatorname{KS}\left(A_{i}\right)}_{2^{\rho\left(A_{i}\right)} \text { times }} \sim \underbrace{A_{i} \times \cdots \times A_{i}}_{8 \text { times }}
$$

where " $\sim$ " denotes an isogeny of abelian varieties. By construction $\operatorname{KS}\left(A_{1}\right) \sim \operatorname{KS}\left(A_{2}\right)$ and then $A_{1}^{8} \sim A_{2}^{8}$. In particular, $A_{1}$ and $A_{2}$ are isogenous and this concludes the proof of Theorem 1.2.

To shorten the notation and according to [14], we introduce two equivalence relations:

Definition 4.2. Let $\left(X_{1}, \alpha_{1}\right)$ and $\left(X_{2}, \alpha_{2}\right)$ be twisted K3 or abelian surfaces.

(i) They are D-equivalent if there exists a twisted Fourier-Mukai equivalence

$$
\Phi: \mathrm{D}^{\mathrm{b}}\left(X_{1}, \alpha_{1}\right) \rightarrow \mathrm{D}^{\mathrm{b}}\left(X_{2}, \alpha_{2}\right) .
$$

(ii) They are $T$-equivalent if there exist $B_{i} \in H^{2}\left(X_{i}, \mathbb{Q}\right)$ such that $\alpha_{i}=\kappa_{A_{i}}\left(B_{i}\right)$ and a Hodge isometry

$$
\varphi: T\left(X_{1}, B_{1}\right) \rightarrow T\left(X_{2}, B_{2}\right)
$$

We can now prove the following easy corollary of Theorem 1.2 :

Corollary 4.3. (i) $\left(\mathrm{Km}\left(A_{1}\right), 1\right)$ is D-equivalent to $\left(\operatorname{Km}\left(A_{2}\right), 1\right)$ if and only if $\left(A_{1}, 1\right)$ and $\left(A_{2}, 1\right)$ are T-equivalent.

(ii) If $\left(A_{1}, \alpha_{1}\right)$ and $\left(A_{2}, \alpha_{2}\right)$ are D-equivalent twisted abelian surfaces, then $\left(\operatorname{Km}\left(A_{1}\right), \Theta_{A_{1}}\left(\alpha_{1}\right)\right)$ and $\left(\operatorname{Km}\left(A_{2}\right), \Theta_{A_{2}}\left(\alpha_{2}\right)\right)$ are D-equivalent.

Proof. Due to the isomorphism in Lemma 4.1, (i) follows trivially from Theorem 1.2, The machinery in [14] applied to the case of abelian surfaces shows that if $\left(A_{1}, \alpha_{1}\right)$ and $\left(A_{2}, \alpha_{2}\right)$ are D-equivalent, then they are T-equivalent as well. Then use Theorem 1.2.

Notice that part (i) of Corollary 4.3 is exactly the analogue of (B) in the introduction.

Remark 4.4. (i) Due to [14, Prop. 8.1], if $\alpha_{j} \in \operatorname{Br}\left(\operatorname{Km}\left(A_{j}\right)\right)$ is non-trivial for any $j \in\{1,2\}$, then the existence of an equivalence $\mathrm{D}^{\mathrm{b}}\left(\operatorname{Km}\left(A_{1}\right), \alpha_{1}\right) \cong \mathrm{D}^{\mathrm{b}}\left(\operatorname{Km}\left(A_{2}\right), \alpha_{2}\right)$ does not imply that $\operatorname{Km}\left(A_{1}\right) \cong \operatorname{Km}\left(A_{2}\right)$ (see also Example 4.3). This is one of the main differences with the untwisted case treated by Hosono, Lian, Oguiso and Yau in [11] (see (A) and (C) in the introduction).

(ii) As suggested by Corollary 4.3, we would expect (ii) in Theorem 1.2 to be equivalent to the existence of a twisted Fourier-Mukai equivalence $\mathrm{D}^{\mathrm{b}}\left(A_{1}, \beta_{1}\right) \cong \mathrm{D}^{\mathrm{b}}\left(A_{2}, \beta_{2}\right)$, where $\beta_{i} \in \operatorname{Br}\left(A_{i}\right)$. This would lead to a twisted version of (A). Actually this is not the case. Indeed, since the period map is surjective for abelian surfaces ([28]), one can produce a counterexample to this expectation by adapting Example 4.11 in [14.

(iii) Let $A_{1}$ and $A_{2}$ be two abelian surfaces with $\mathrm{NS}\left(A_{1}\right)=\left\langle H_{1}\right\rangle$ and $\mathrm{NS}\left(A_{2}\right)=\left\langle H_{2}\right\rangle$. If there exist $\alpha_{1} \in \operatorname{Br}\left(\operatorname{Km}\left(A_{1}\right)\right)$ and $\alpha_{2} \in \operatorname{Br}\left(\operatorname{Km}\left(A_{2}\right)\right)$ such that $\mathrm{D}^{\mathrm{b}}\left(\operatorname{Km}\left(A_{1}\right), \alpha_{1}\right) \cong \mathrm{D}^{\mathrm{b}}\left(\operatorname{Km}\left(A_{2}\right), \alpha_{2}\right)$ then $H_{1}^{2} / H_{2}^{2}$ is a square in $\mathbb{Q}$. Indeed, by Theorem 1.2 (and by [14, Sect. 7]), if $\mathrm{D}^{\mathrm{b}}\left(\operatorname{Km}\left(A_{1}\right), \alpha_{1}\right) \cong$ $\mathrm{D}^{\mathrm{b}}\left(\operatorname{Km}\left(A_{2}\right), \alpha_{2}\right)$ then there exists an isogeny $\varphi: A_{1} \rightarrow A_{2}$ inducing a Hodge isometry $\varphi^{*}$ : $H^{2}\left(A_{2}, \mathbb{Q}\right) \rightarrow H^{2}\left(A_{1}, \mathbb{Q}\right)$ such that $\varphi^{*}\left(H_{2}\right)=q H_{1}$, for some $q \in \mathbb{Q}$. In particular $H_{2}^{2}=q^{2} H_{1}^{2}$.

4.3. An explicit example. In this example, we use Theorem 1.2 to establish a connection between the twisted derived categories of some nice Kummer surfaces with Picard number 2. Recall that the lattices $U$ and $U(n)$ are the free abelian group $\mathbb{Z} \oplus \mathbb{Z}$ endowed respectively with the quadratic forms represented by the matrices

$$
\left(\begin{array}{ll}
0 & 1 \\
1 & 0
\end{array}\right) \text { and }\left(\begin{array}{ll}
0 & n \\
n & 0
\end{array}\right)
$$


Let $A$ be an abelian surface such that $\operatorname{NS}(A) \cong U(n)$, for some positive integer $n$. We first show that there exist two elliptic curves $E$ and $F$ and a subgroup $C_{n} \cong \mathbb{Z} / n \mathbb{Z}$ of $E \times F$ such that either $A \cong(E \times F) / C_{n}$ or $\widehat{A} \cong(E \times F) / C_{n}$.

To see this, let us first observe that, since $\mathrm{NS}(A) \cong U(n)$, the transcendental lattice $T(A)$ is isometric to $U(n) \oplus U$. Indeed for any abelian surface $A, H^{2}(A, \mathbb{Z})$, endowed with the cup-product, is isometric to the lattice $U \oplus U \oplus U$ (see [17] for more details).

We choose a basis $\left\langle e_{1}, e_{2}, f_{1}, f_{2}\right\rangle=U \oplus U(n) \hookrightarrow U^{3}$, an isometry $\varphi: H^{2}(A, \mathbb{Z}) \rightarrow U^{3}$ and $c \in \mathbb{C}$ such that

$$
\varphi\left(c \sigma_{A}\right)=e_{1}-n \omega_{1} \omega_{2} e_{2}+\omega_{1} f_{1}+\omega_{2} f_{2},
$$

where $H^{2,0}(A)=\left\langle\sigma_{A}\right\rangle$. We define in $\mathbb{C}$ the lattices $\Gamma_{1}=\mathbb{Z}+\omega_{1} \mathbb{Z}$ and $\Gamma_{2}=\mathbb{Z}+\omega_{2} \mathbb{Z}$ and the elliptic curves $E:=\mathbb{C} / \Gamma_{1}$ and $F:=\mathbb{C} / \Gamma_{2}$. Notice that, since $T(A) \cong U(n) \oplus U$ and $\sigma_{A}^{2}=0$, the numbers $1, \omega_{1}, \omega_{2}$ and $\omega_{1} \omega_{2}$ are linearly independent over $\mathbb{Q}$. So, in particular, $E$ and $F$ are not isogenous. If $H_{1}(E \times F, \mathbb{Z})=\left\langle\gamma_{1}, \gamma_{2}, \delta_{1}, \delta_{2}\right\rangle$, then we consider the subgroup $C_{n}$ of $E \times F$ such that

$$
H_{1}\left((E \times F) / C_{n}, \mathbb{Z}\right)=\left\langle\frac{\gamma_{1}+\delta_{1}}{n}, \gamma_{2}, \delta_{1}, \delta_{2}\right\rangle .
$$

Let $S:=(E \times F) / C_{n}$. In terms of the dual bases of the bases of $H_{1}(E \times F, \mathbb{Z})$ and $H_{1}(S, \mathbb{Z})$ just described, we write $H^{1}(S, \mathbb{Z})=\left\langle\mathrm{d} z_{1}, \mathrm{~d} z_{2}, \mathrm{~d} w_{1}, \mathrm{~d} w_{2}\right\rangle$ and $H^{1}(E \times F, \mathbb{Z})=\left\langle\mathrm{d} x_{1}, \mathrm{~d} x_{2}, \mathrm{~d} y_{1}, \mathrm{~d} y_{2}\right\rangle$. If $\pi: E \times F \rightarrow S$ is the natural surjection, the map $\theta:=\pi^{*}: H^{1}(S, \mathbb{Z}) \rightarrow H^{1}(E \times F, \mathbb{Z})$ is such that:

$$
\begin{array}{lll}
\theta\left(\mathrm{d} z_{1}\right)=n \mathrm{~d} x_{1}, & \theta\left(\mathrm{d} w_{1}\right)=-\mathrm{d} x_{1}+\mathrm{d} y_{1}, \\
\theta\left(\mathrm{d} z_{2}\right)=\mathrm{d} x_{2}, & \theta\left(\mathrm{d} w_{2}\right)=\mathrm{d} y_{2} .
\end{array}
$$

Observe that $\operatorname{NS}(E \times F)=\left\langle\mathrm{d} x_{1} \wedge \mathrm{d} x_{2}, \mathrm{~d} y_{1} \wedge \mathrm{d} y_{2}\right\rangle$. Furthermore, due to the properties in (4.6) which characterize the morphism $\stackrel{2}{\wedge}^{2}: H^{2}(S, \mathbb{Z}) \rightarrow H^{2}(E \times F, \mathbb{Z})$ and due to the fact that $\wedge^{2} \theta$ preserves the Hodge structures on $H^{2}(S, \mathbb{Z})$ and $H^{2}(E \times F, \mathbb{Z})$,

$$
\begin{aligned}
\mathrm{NS}(S) & =\left\langle\mathrm{d} z_{1} \wedge \mathrm{d} z_{2}, n \mathrm{~d} w_{1} \wedge \mathrm{d} w_{2}+\mathrm{d} z_{1} \wedge \mathrm{d} w_{2}\right\rangle, \\
T(S) & =\left\langle\mathrm{d} z_{1} \wedge \mathrm{d} w_{1}, \mathrm{~d} z_{2} \wedge \mathrm{d} w_{2}, \mathrm{~d} z_{1} \wedge \mathrm{d} w_{2},-n \mathrm{~d} w_{1} \wedge \mathrm{d} z_{2}+\mathrm{d} z_{1} \wedge \mathrm{d} w_{2}\right\rangle .
\end{aligned}
$$

In particular, $\mathrm{NS}(S) \cong U(n)$ and $T(S) \cong U \oplus U(n)$.

Consider the two cohomology classes

$$
\begin{aligned}
\sigma_{E \times F} & =\mathrm{d} x_{1} \wedge \mathrm{d} y_{1}+\omega_{2} \mathrm{~d} x_{1} \wedge \mathrm{d} y_{2}+\omega_{1} \mathrm{~d} x_{2} \wedge \mathrm{d} y_{1}+\omega_{1} \omega_{2} \mathrm{~d} x_{2} \wedge \mathrm{d} y_{2} \\
\sigma_{S} & =\mathrm{d} z_{1} \wedge \mathrm{d} w_{1}+\omega_{2} \mathrm{~d} z_{1} \wedge \mathrm{d} w_{2}+\omega_{1}\left(n \mathrm{~d} w_{1} \wedge \mathrm{d} z_{2}-\mathrm{d} z_{1} \wedge \mathrm{d} w_{2}\right)+n \omega_{1} \omega_{2} \mathrm{~d} z_{2} \wedge \mathrm{d} w_{2} .
\end{aligned}
$$

Obviously, $\sigma_{E \times F} \in T(E \times F) \otimes \mathbb{C}$ and $\sigma_{S} \in T(S) \otimes \mathbb{C}$. Since $\left\langle\sigma_{E \times F}\right\rangle=H^{2,0}(E \times F)$ and since an easy calculation shows that $\stackrel{2}{\wedge} \theta\left(\sigma_{S}\right)=n \sigma_{E \times F},\left\langle\sigma_{S}\right\rangle=H^{2,0}(S)$. This implies that, due to (4.5), there exists an isometry $\eta: H^{2}(S, \mathbb{Z}) \rightarrow U^{3}$ such that $\eta^{-1} \circ \varphi: H^{2}(A, \mathbb{Z}) \rightarrow H^{2}(S, \mathbb{Z})$ is a Hodge isometry (see [28]). The Torelli Theorem for abelian surfaces shows that either $A \cong(E \times F) / C_{n}$ or $\widehat{A} \cong(E \times F) / C_{n}$.

Observe that, since $\mathrm{NS}(A) \cong U(n)$, the abelian surface $A$ is principally polarized if and only if $n=1$. This means that, if $n \neq 1,(E \times F) / C_{n}$ and its dual are not isomorphic. Furthermore, $A$ and $E \times F$ are isogenous but $T(A) \neq T(E \times F)$. Therefore, due to Theorem 2.2, $\mathrm{D}^{\mathrm{b}}(A) \neq$ $\mathrm{D}^{\mathrm{b}}(E \times F)$. This proves that there are isogenous abelian surfaces whose derived categories of (untwisted) coherent sheaves are not equivalent (see Remark 3.4).

Choose the standard basis $\left\{g_{1}, g_{2}, k_{1}, k_{2}\right\}$ for $U \oplus U$. Due to the explicit description of $T(A)$ that we have previously given, it is straightforward to see that there exists an inclusion $i_{1}: T(A) \rightarrow U \oplus U$ where $i_{1}\left(e_{j}\right)=g_{j}(j \in\{1,2\}), i_{1}\left(f_{1}\right)=n k_{1}$ and $i_{1}\left(f_{2}\right)=n k_{2}$. Let $\sigma:=i\left(\sigma_{A}\right) \in U^{2} \otimes \mathbb{C}$. Due to (4.5), we can write $\sigma=g_{1}-n \omega_{1} \omega_{2} g_{2}+n \omega_{1} h_{1}+\omega_{2} h_{2}$.

Consider in $\mathbb{C}$ the lattice $\Gamma_{3}=\mathbb{Z}+n \omega_{1} \mathbb{Z}$ and the elliptic curve $E_{1}:=\mathbb{C} / \Gamma_{3}$. Of course, $E$ and $E_{1}$ are isogenous. Reasoning as before and using the surjectivity of the period map and the Torelli 
Theorem for abelian surfaces ([28]), we get an isometry $\varphi_{1}: T\left(E_{1} \times F\right) \rightarrow U^{2}$ fitting in the following commutative diagram:

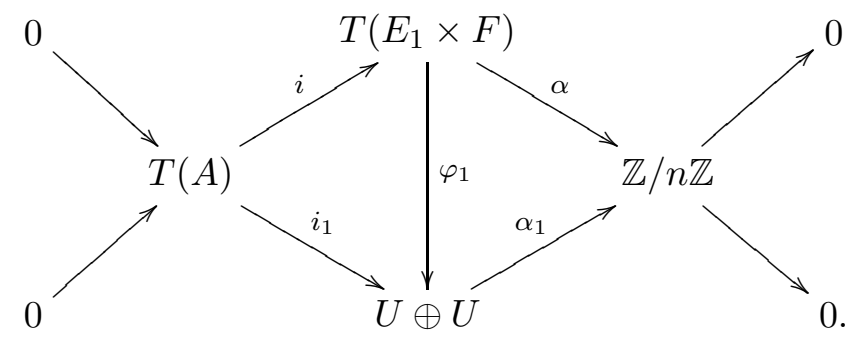

Of course, $i$ preserves the Hodge structures and $\alpha \in \operatorname{Br}\left(E_{1} \times F\right)$. Proposition 4.7 in [12] yields $B \in H^{2}(E \times F, \mathbb{Q})$ such that $(A, 1)$ and $\left(E \times F, \kappa_{E \times F}(B)\right)$ are T-equivalent. By Theorem 1.2, there exist $\beta \in \operatorname{Br}\left(\operatorname{Km}\left(E_{1} \times F\right)\right)$ of order $n$ and a twisted Fourier-Mukai equivalence $\mathrm{D}^{\mathrm{b}}(\operatorname{Km}(A)) \cong$ $\mathrm{D}^{\mathrm{b}}\left(\operatorname{Km}\left(E_{1} \times F\right), \beta\right)$.

4.4. The number of twisted Kummer structures. As an easy corollary of Lemma 4.1, we get a surjective map

$\Psi:\{$ Twisted abelian surfaces $\} /$ isom $\longrightarrow\{$ Twisted Kummer surfaces $\} /$ isom

which sends the isomorphism class $[(A, \alpha)]$ to the isomorphism class $\left[\left(\operatorname{Km}(A), \Theta_{A}(\alpha)\right)\right]$. The main result in [11] proves that the preimage of $[(\operatorname{Km}(A), 1)]$ is finite, for any abelian surface $A$ and $1 \in \operatorname{Br}(A)$ the trivial class (see [11, Thm. 0.1]). On the other hand [11] shows that the cardinality of the preimages of $\Psi$ can be arbitrarily large. This answered an old question by Shioda. Namely, there can be many non-isomorphic (untwisted) abelian surfaces giving rise to isomorphic (untwisted) Kummer surfaces (a partial result in this direction is also contained in [10]). This is usually rephrased saying that on a K3 surface one can put many non-isomorphic (untwisted) Kummer structures.

Using Theorem 1.2, the picture in [11] can be completely generalized to the twisted case.

Proposition 4.5. (i) For any twisted Kummer surface $(\operatorname{Km}(A), \alpha)$, the preimage $\Psi^{-1}([(\operatorname{Km}(A), \alpha)])$ is finite.

(ii) For positive integers $N$ and $n$, there exists a twisted Kummer surface $(\operatorname{Km}(A), \alpha)$ with $\alpha$ of order $n$ in $\operatorname{Br}(\operatorname{Km}(A))$ and such that $\left|\Psi^{-1}([(\operatorname{Km}(A), \alpha)])\right| \geq N$.

Proof. Suppose that $\Psi\left(\left[\left(A_{1}, \alpha_{1}\right)\right]\right)=\Psi\left(\left[\left(A_{2}, \alpha_{2}\right)\right]\right)=[(\operatorname{Km}(A), \alpha)]$, i.e. suppose that there exists an isomorphism $f: \operatorname{Km}(A) \stackrel{\sim}{\rightarrow} \operatorname{Km}\left(A_{i}\right)$ such that $f^{*} \Theta_{A_{i}}\left(\alpha_{i}\right)=\alpha$. In particular,

$$
\mathrm{D}^{\mathrm{b}}\left(\operatorname{Km}\left(A_{1}\right), \Theta_{A_{1}}\left(\alpha_{1}\right)\right) \cong \mathrm{D}^{\mathrm{b}}\left(\operatorname{Km}\left(A_{2}\right), \Theta_{A_{2}}\left(\alpha_{2}\right)\right) .
$$

Due to Theorem 1.2, the proof of (i) amounts to show that, up to isomorphisms, there are finitely many T-equivalent twisted abelian surfaces $\left(A^{\prime}, \beta\right)$ such that $\Psi\left(\left[\left(A^{\prime}, \beta\right)\right]\right)=[(\operatorname{Km}(A), \alpha)]$. Since, up to isomorphisms, there are just finitely many abelian surfaces $A^{\prime}$ with $\mathrm{D}^{\mathrm{b}}\left(A^{\prime}\right) \cong \mathrm{D}^{\mathrm{b}}(A)$ ([3] , Prop. 5.3]), we can just fix $A^{\prime}$ with such a property and show that, up to isomorphisms, there exists a finite number of $\beta^{\prime} \in \operatorname{Br}\left(A^{\prime}\right)$ such that $\left(A^{\prime}, \beta\right)$ and $\left(A^{\prime}, \beta^{\prime}\right)$ are T-equivalent. But this is the content of [14, Prop. 3.4] for the case of abelian surfaces.

Applying the results in [24] and 29] to abelian surfaces, we see that, for any positive integer $N$, there exist $N$ non-isomorphic abelian surfaces $A_{1}, \ldots, A_{N}$ such that $\mathrm{D}^{\mathrm{b}}\left(A_{i}\right) \cong \mathrm{D}^{\mathrm{b}}\left(A_{j}\right)(i, j \in$ $\{1, \ldots, N\})$. Due to Theorem 2.2, for any $i \in\{2, \ldots, N\}$, there is a Hodge isometry

$$
g_{i}: T\left(A_{1}\right) \rightarrow T\left(A_{i}\right) \text {. }
$$

Take $B_{1} \in T\left(A_{1}\right) \otimes \mathbb{Q}$ such that $\alpha_{1}:=\kappa_{A_{1}}\left(B_{1}\right)$ and $\Theta_{A_{1}}\left(\alpha_{1}\right)$ are not trivial in $\operatorname{Br}\left(A_{1}\right)$ and $\operatorname{Br}\left(\operatorname{Km}\left(A_{1}\right)\right)$ respectively. We can also choose $\alpha_{1}$ such that the order of $\Theta_{A_{1}}\left(\alpha_{1}\right)$ is $n$ in $\operatorname{Br}\left(\operatorname{Km}\left(A_{1}\right)\right)$. 
Then, for any $i \in\{2, \ldots, N\}$, define $\alpha_{i}:=\kappa_{A_{i}}\left(g_{i}\left(B_{1}\right)\right)$. Obviously, $\left(A_{i}, \alpha_{i}\right)$ and $\left(A_{j}, \alpha_{j}\right)$ are Tequivalent when $i, j \in\{1, \ldots, N\}$. Theorem 1.2 immediately implies that $\left(\operatorname{Km}\left(A_{i}\right), \Theta_{A_{i}}\left(\alpha_{i}\right)\right)$ and $\left(\operatorname{Km}\left(A_{j}\right), \Theta_{A_{j}}\left(\alpha_{j}\right)\right)$ are D-equivalent.

For any $i \in\{2, \ldots, N\}$, the isometry $g_{i}$ induces a Hodge isometry $f_{i}: T\left(\operatorname{Km}\left(A_{1}\right)\right) \rightarrow T\left(\operatorname{Km}\left(A_{i}\right)\right)$ which (due to [23, Thm. 1.14.4]) extends to a Hodge isometry $h_{i}: H^{2}\left(\operatorname{Km}\left(A_{1}\right), \mathbb{Z}\right) \rightarrow H^{2}\left(\operatorname{Km}\left(A_{i}\right), \mathbb{Z}\right)$. The Torelli Theorem yields an isomorphism $\varphi_{i}: \operatorname{Km}\left(A_{1}\right) \rightarrow \operatorname{Km}\left(A_{i}\right)$ such that $\varphi_{i}^{*}\left(\Theta_{A_{i}}\left(\alpha_{i}\right)\right)=$ $\Theta_{A_{1}}\left(\alpha_{1}\right)$ (possibly changing $\alpha_{i}$ with $\alpha_{i}^{-1}$ ), for any $i \in\{2, \ldots, N\}$. This concludes the proof of (ii).

In other words, Proposition 4.5 shows that on a twisted K3 surface we can put just a finite number of non-isomorphic twisted Kummer structures. Nevertheless, such a number can be arbitrarily large even when the twist is non-trivial and has any possible order.

Acknowledgements. The final version of this paper was written during the author stay at the MaxPlanck-Institut für Mathematik (Bonn). The results in this paper benefit of many interesting discussions with Daniel Huybrechts and Bert van Geemen. It is a great pleasure to thank them for their help and suggestions.

\section{REFERENCES}

[1] A. Beauville, Variétés Kähleriennes dont la première classe de Chern est nulle, J. Diff. Geom. 18 (1983), 755782 .

[2] T. Bridgeland, A. King, M. Reid, The MacKay correspondence as an equivalence of derived categories, J. Am. Math. Soc. 14 (2001), 535-554.

[3] T. Bridgeland, A. Maciocia, Complex surfaces with equivalent derived categories, Math. Z. 236 (2001), 677-697.

[4] A. Căldăraru, Derived categories of twisted sheaves on Calabi-Yau manifolds, Ph.D.-Thesis, Cornell University (2002).

[5] A. Căldăraru, The Mukai pairing, II: the Hochschild-Kostant-Rosenberg isomorphism, Adv. Math. 194 (2005), $34-66$.

[6] A. Canonaco, P. Stellari, Twisted Fourier-Mukai functors, to appear in: Adv. Math., math.AG/0605229

[7] A.J. de Jong, A result of Gabber, preprint (2003).

[8] V. Golyshev, V. Lunts, D. Orlov, Mirror symmetry for abelian varieties, J. Algebraic Geom. 10 (2001), $433-496$.

[9] T. Gómez, Algebraic stacks, Proc. Indian Acad. Sci. Math. Sci. 111 (2001), 1-31.

[10] V. Gritsenko, K. Hulek, Minimal Siegel modular threefolds, Math. Proc. Camb. Phil. Soc. 123 (1998), $461-485$.

[11] S. Hosono, B.H. Lian, K. Oguiso, S.-T. Yau, Kummer structures on a K3 surface - An old question of T. Shioda, Duke Math. J. 12 (2003), 635-647.

[12] D. Huybrechts, Generalized Calabi-Yau structures, K3 surfaces, and B-fields, Int. J. Math. 16 (2005), 13-36.

[13] D. Huybrechts, Fourier-Mukai transforms in algebraic geometry, Oxford Mathematical Monographs, Oxford Science Publications (2006).

[14] D. Huybrechts, P. Stellari, Equivalences of twisted K3 surfaces, Math. Ann. 332 (2005), 901-936.

[15] D. Huybrechts, P. Stellari, Proof of Căldăraru's conjecture, to appear in: The 13th MSJ Inter. Research Inst. Moduli Spaces and Arithmetic Geometry, Adv. Stud. Pure Math., AMS, math.AG/0411541

[16] Y. Kawamata, Equivalences of derived categories of sheaves on smooth stacks, Am. J. Math. 126 (2004), 10571083.

[17] D.R. Morrison, On K3 surfaces with large Picard number, Invent. Math. 75 (1984), 105-121.

[18] D.R. Morrison, The Kuga-Satake variety of an abelian suface, J. Algebra 92 (1985), 454-476.

[19] S. Mukai, Semi-homogeneous vector bundles on an abelian variety, J. Math. Kyoto Univ. 18 (1978), $239-272$.

[20] S. Mukai, On the moduli space of bundles on K3 surfaces, I, In: Vector Bundles on Algebraic Varieties, Oxford University Press, Bombay and London (1987), 341-413.

[21] Y. Namikawa, Counter-example to global Torelli problem for irreducible symplectic manifolds, Math. Ann. 324 (2002), 841-845.

[22] V.V. Nikulin, On Kummer surfaces, Math. USSR Izvestija 9 (1975), 261-275.

[23] V.V. Nikulin, Integral symmetric bilinear forms and some of their applications, Math. USSR Izvestija 14 (1980), $103-167$.

[24] K. Oguiso, K3 surfaces via almost-primes, Math. Research Letters 9 (2002), 47-63.

[25] D. Orlov, Equivalences of derived categories and K3 surfaces, J. Math. Sci. 84 (1997), 1361-1381.

[26] D. Orlov, Derived categories of coherent sheaves on abelian varieties and equivalences between them, Izv. Math. 66 (2002), 569-594. 
[27] D. Ploog, Equivariant autoequivalences for finite group actions, math.AG/0508625.

[28] T. Shioda, The period map of abelian surfaces, J. Fac. Sci. Univ. Tokyo 25 (1978), 45-59.

[29] P. Stellari, Some remarks about the FM-partners of K3 surfaces with Picard numbers 1 and 2, Geom. Dedicata 108 (2004), 1-13.

[30] K. Yoshioka, Moduli spaces of stable sheaves on abelian surfaces, Math. Ann. 321 (2001), 817-884.

Dipartimento di Matematica "F. Enriques", Università degli Studi di Milano, Via Cesare Saldini 50, 20133 Milano, Italy

E-mail address: stellari@mat.unimi.it 